\title{
Evading the Lyth bound in hybrid natural inflation
}

\author{
A. Hebecker, ${ }^{1, *}$ S. C. Kraus, ${ }^{1, \dagger}$ and A. Westphal ${ }^{2,3, *}$ \\ ${ }^{1}$ Institute for Theoretical Physics, Heidelberg University, D-69120, Germany \\ ${ }^{2}$ Deutsches Elektronen-Synchrotron DESY, Theory Group, D-22603 Hamburg, Germany \\ ${ }^{3}$ Kavli Institute for Theoretical Physics, Santa Barbara, California 93106, USA
}

(Received 31 May 2013; published 2 December 2013)

\begin{abstract}
Generically, the gravitational-wave or tensor-mode contribution to the primordial curvature spectrum of inflation is tiny if the field range of the inflaton is much smaller than the Planck scale. We show that this pessimistic conclusion is naturally avoided in a rather broad class of small-field models. More specifically, we consider models where an axionlike shift symmetry keeps the inflaton potential flat (up to nonperturbative cosine-shaped modulations), but inflation nevertheless ends in a waterfall regime, as is typical for hybrid inflation. In such hybrid natural inflation scenarios (examples are provided by Wilson line inflation and fluxbrane inflation), the slow-roll parameter $\epsilon$ can be sizable during an early period (relevant for the cosmic microwave background spectrum). Subsequently, $\epsilon$ quickly becomes very small before the tachyonic instability eventually terminates the slow-roll regime. In this scenario, one naturally generates a considerable tensor-mode contribution in the curvature spectrum, collecting nevertheless the required amount of $e$-foldings during the final period of inflation. While nonobservation of tensors by Planck is certainly not a problem, a discovery in the medium- to long-term future is realistic.
\end{abstract}

DOI: 10.1103/PhysRevD.88.123506

PACS numbers: $98.80 . \mathrm{Cq}, 11.25 . \mathrm{Wx}, 14.80 . \mathrm{Va}$

\section{INTRODUCTION}

Cosmological inflation, and in particular the generation of curvature perturbations through quantum fluctuations of the inflaton field, allows for a surprisingly accurate description of recent Planck data [1,2]. Concrete fieldtheoretic realizations roughly fall in two classes: large-field and small-field models.

In the first class, the canonically normalized inflaton field $\phi$ covers a large distance in field space, $\Delta \phi \gg M_{P}$, during the last $60 e$-folds. This has the advantage that it can be realized with a very simple potential, e.g., of the form $\sim \phi^{n}$ [3]. In addition, such models have the very interesting feature of producing sizable tensor perturbations without fine-tuning. However, they require the absence or unnatural smallness of all higher-dimension operators $\sim \phi^{m}$ with $m>n$, which is unnatural unless an approximate shift symmetry $\phi \rightarrow \phi+c$ is present.

By contrast, small-field models work with $\Delta \phi \ll M_{P}$, but they require a nongeneric, flat potential. Here flatness means in particular that, in spite of a significant constant term in the potential, the coefficients of the $\phi$ and $\phi^{2}$ terms have to be unnaturally small. In principle, this can be easily realized either by explicit tuning of the dimension-six operators [4-7] or using a shift symmetry as in natural inflation ${ }^{1}$ [9], $\phi \rightarrow \phi+c$. However, for inflation to end, sizable interactions of $\phi$ have to be present in some region

\footnotetext{
*A.Hebecker@ThPhys.Uni-Heidelberg.de

†S.Kraus@ThPhys.Uni-Heidelberg.de

¥Alexander.Westphal@desy.de

${ }^{1}$ A closely related proposal is Wilson line (or "extranatural") inflation [8].
}

in field space, which nevertheless leads to fine-tuning. ${ }^{2}$ This discussion can be taken to the more advanced level of supergravity, where it takes the form of arguing about natural sizes of higher-order terms in the Kähler potential $[11,12]$. Most importantly for us, additional fine-tuning is necessary to obtain large tensor perturbations.

We believe that the discussion about natural or unnatural sizes of operator coefficients can (at least in principle) be more fruitful in the context of a UV complete model, the natural candidate being of course superstring theory. Constructing large-field models in string theory has been notoriously difficult for some time. However, recently considerable progress took place based on constructions of (axion) monodromy and unwinding inflation; see, e.g., Refs. [13-22]. Here we focus on small-field models. As we will argue, there is an interesting class of stringy (or at least stringinspired) models, where a shift symmetry guarantees the flatness of the potential, and tensor perturbations can be sizable in spite of the small field range.

Before describing such stringy constructions, we recall the Lyth bound and our general plan for avoiding it: In slow-roll inflation, the tensor-to-scalar ratio (i.e., the ratio of the gravitational-wave power spectrum $\Delta_{\mathcal{T}}^{2} \sim H^{2}$ and the scalar power spectrum $\Delta_{\mathcal{R}}^{2} \sim H^{2} / \epsilon$ ) is proportional to the first slow-roll parameter, $r=\Delta_{\mathcal{T}}^{2} / \Delta_{\mathcal{R}}^{2}=16 \epsilon$. (We use $M_{P}=1$ here and below.) This can be rewritten in terms of the field-variation per $e$-fold as

\footnotetext{
${ }^{2}$ In addition to these shift symmetry-breaking interactions, the inflaton can couple shift-symmetrically to gauge fields. This coupling may provide an efficient channel for reheating [10].
} 


$$
\sqrt{\frac{r}{8}} \simeq\left|\frac{\mathrm{d} \phi}{\mathrm{d} N}\right| .
$$

Thus, if $r$ is roughly constant or monotonically increasing during inflation, the size of tensor modes produced within the initial observable $10 e$-folds of the cosmologically needed $60 e$-folds of inflation is bounded by the total field excursion during these $60 e$-folds $[23,24] .^{3}$ In particular, for small-field inflation $r$ is typically negligibly small.

In many small-field models, inflation ends because the evolution of the inflaton smoothly changes from slow-roll to fast-roll. In this sense, a monotonically increasing $\epsilon$ is a common feature. However, there are certainly exceptions: For example, if inflation ends in a "waterfall" classical instability of a second scalar field, as in hybrid inflation ${ }^{4}$ [36,37], $\epsilon$ can decrease monotonically, allowing for a large tensor signal during the observable $10 e$-folds.

Combining the concept of a shift symmetry, protecting the flatness of the potential against radiative corrections, with the idea of hybrid inflation leads to hybrid natural inflation $^{5}$ [38-44]. The shift symmetry is generically broken by nonperturbative effects, inducing a periodic cosine potential for the axion. This is a rather attractive setting, as it overcomes the problematic issue [45] of a super-Planckian axion decay constant in natural inflation (see also Refs. [16,46-48]). If, in such a model, the waterfall sets in close to the minimum of the potential, $\epsilon$ can be sizable during the observable $e$-folds of inflation, while the bulk of the required $60 e$-folds is accumulated at later stages, when $\epsilon$ is very small. This quite naturally provides us with a potentially detectable tensor signal, in spite of the small field range.

Motivated by the above considerations, we now focus on string theory realizations of hybrid natural inflation. They arise as models of $D$-term hybrid inflation $[49,50]$ and can be more specifically characterized as Wilson line inflation [44] on the type IIA or as fluxbrane inflation [51,52] on the (mirror dual) type IIB side. We take the type IIB (or fluxbrane) point of view because moduli stabilization is better

\footnotetext{
${ }^{3}$ The bound arising from a situation of monotonically increasing $\epsilon$ during the last $60 e$-folds of inflation is considered in Ref. [24]. The original paper [23] only takes into account the field excursion during the observable $\sim 10 e$-folds.

${ }^{4}$ Supersymmetric hybrid inflation incorporating various corrections was investigated in the minimal supersymmetric hybrid inflation program $[25,26]$, where it was found that, in some regions of parameter space, this model can produce sizable gravity waves [27]. More generally, large tensor signals in small-field inflation can be obtained whenever a sufficiently complicated potential is tuned in order to achieve a nonmonotonic evolution of $\epsilon$ (see, for example, Refs. [28,29]). Other suggestions for avoiding the Lyth bound and related constraints are discussed, e.g., in Refs. [30-35].

${ }^{5}$ The inflaton can be a pseudo-Nambu-Goldstone boson [38-42] or a Wilson line $[42,43]$. The proposed models go by various names, such as "little inflatons" or "pseudonatural inflation." Wilson line inflation was put into a stringy context in Ref. [44].
}

understood in this setting and because of the more intuitive, geometric picture of inflation; in this scenario, the inflaton is the transverse separation of a pair of D7-branes. Since the two branes carry opposite gauge flux, they move toward each other, which corresponds to the slow rolling of the inflaton. At a certain (extremely small) critical distance, the gauge flux annihilates. This is the waterfall regime, with the waterfall fields realized by open strings stretched between the branes. The presence of a shift symmetry can be most easily understood via T-duality to type IIA: The duality maps the brane deformation modulus to a Wilson line, which is known to enjoy a shift symmetry at large volume. This shift symmetry is broken by nonperturbative effects, giving rise to the periodic (cosine shaped) potential alluded to above.

For avoiding the Lyth bound, it is essential that the waterfall sets in very close to the minimum of the potential. From a purely field-theoretic perspective, this requires some tuning of Lagrangian parameters or an appropriate model building effort. ${ }^{6}$ From the fluxbrane perspective, however, things look different: The energy of the twobrane system is minimized in a situation where the branes come very close to each other. This is where the waterfall sets in. Moreover, while the maximal possible field excursion corresponds to roughly the length scale of the compact manifold, the critical distance of the waterfall instability is substringy [51,53]. Thus, a large hierarchy between the maximal and the critical brane-to-brane distance is natural.

In this paper we discuss the phenomenology of a general hybrid inflation model with a periodic potential. In this model a sizable tensor-to-scalar ratio can be obtained for a Planckian axion decay constant. However, stringy consistency conditions dictate bounds on those decay constants. The examples of Kähler and complex structure axions are examined. The latter is of particular interest for us as, from an F-theory perspective, the inflaton, being a D7-brane deformation modulus, is part of the complex structure moduli space of the fourfold. We argue that for generic values of the complex structure, the tensor-to-scalar ratio can be as large as $r \sim 10^{-3}$.

\section{EFFECTIVE POTENTIAL OF FLUXBRANE INFLATION}

The effective scalar potential of fluxbrane inflation, or its T-dual cousin Wilson line inflation, can be approximated by a hybrid inflation setup with a periodic inflaton dependence from a nonperturbative correction. With $\phi, \chi$ denoting the canonically normalized inflaton and waterfall field, respectively, we have

\footnotetext{
${ }^{6}$ For example, the authors of Refs. [40,41] find that inflation most naturally starts and ends above the inflection point of the potential. In this regime $\epsilon$ increases monotonically, and thus the Lyth bound applies, leading to a small tensor-to-scalar ratio. By contrast, the authors of Ref. [39] achieve waterfall near the minimum through field theory model building, but they do not consider tensor modes.
} 
$V(\phi, \chi)=\frac{\lambda}{4}\left(\chi^{2}-\frac{2 \Lambda^{2}}{\sqrt{\lambda}}\right)^{2} \cdot\left[1-\alpha \cos \left(\frac{\phi}{f}\right)\right]+g \phi^{2} \chi^{2}$.

Inflation is driven by $\phi$ in slow-roll at

$$
\phi>\phi_{c}, \quad \phi_{c}^{2}=\frac{\sqrt{\lambda}}{g} \Lambda^{2},
$$

with the vacuum energy dominantly sitting in the first term of Eq. (2) at $\chi=0$. This entails $\alpha<1$. (We choose $\alpha>0$ by convention.) Inflation ends via a waterfall instability in $\chi$ once $\phi<\phi_{c}$. Keeping the vacuum energy $\Lambda^{4}$ during inflation fixed, we can adjust $\phi_{c} \ll 1$ as small as we like by choosing appropriately $\sqrt{\lambda} \ll g<1$.

During inflation at $\phi>\phi_{c}$, the dynamics is therefore governed by the effective potential

$$
V(\phi)=\Lambda^{4}\left[1-\alpha \cos \left(\frac{\phi}{f}\right)\right] .
$$

The slow-roll parameters are [40-42]

$$
\begin{aligned}
\epsilon & =\frac{1}{2}\left(\frac{V^{\prime}}{V}\right)^{2}=\frac{\alpha^{2}}{2 f^{2}} \frac{\sin ^{2}(\phi / f)}{(1-\alpha \cos (\phi / f))^{2}}, \\
\eta & =\frac{V^{\prime \prime}}{V}=\frac{\alpha}{f^{2}} \frac{\cos (\phi / f)}{1-\alpha \cos (\phi / f)}, \\
\xi^{2} & =-\frac{V^{\prime} V^{\prime \prime \prime}}{V^{2}}=\frac{\alpha^{2}}{f^{4}} \frac{\sin ^{2}(\phi / f)}{(1-\alpha \cos (\phi / f))^{2}},
\end{aligned}
$$

from which the two-point function observables can be computed as

$$
\begin{aligned}
n_{S} & =1-6 \epsilon+2 \eta, \quad r=16 \epsilon, \\
\frac{\mathrm{d} n_{S}}{\mathrm{~d} \ln k} & =16 \epsilon \eta-24 \epsilon^{2}+2 \xi^{2} .
\end{aligned}
$$

We see from Eq. (5) that, for a given potential, maximizing $r$ means taking $\eta \rightarrow 0$. Hence, in a first step, we choose $\phi_{N}=\phi_{0} \equiv \pi f / 2$ to be the fixed starting point as there we have $V^{\prime}$ maximal while $V^{\prime \prime}=0$.

We can now compute the number of $e$-folds $N$ elapsing between the initial field value $\phi_{N}=\phi_{0}$ and the final value $\phi_{c}$ in the limit $\phi_{c} / f \ll 1$ :

$$
N=\int_{t\left(\phi_{c}\right)}^{t\left(\phi_{0}\right)} \mathrm{d} t H=\int_{\phi_{c}}^{\phi_{0}} \frac{\mathrm{d} \phi}{\sqrt{2 \epsilon}} \simeq \frac{f^{2}}{\alpha} \ln \left(\frac{4 \phi_{0}}{\pi \phi_{c}}\right) .
$$

This can be dialed by choosing the waterfall exit $\phi_{c}$ appropriately, so that $N=60$, i.e.,

$$
\phi_{c} \simeq \frac{4}{\pi} \phi_{0} \exp \left(-\frac{60 \alpha}{f^{2}}\right) .
$$

As $\phi_{0}$ is now the point at $60 e$-folds before the end of inflation, the observables at CMB scales are evaluated at $\phi=\phi_{0}$. This gives the two-point function observables

$$
\begin{aligned}
n_{S} & \simeq 1-6 \epsilon=1-\frac{3}{8} r \simeq 0.962+0.038(1-r / 0.1), \\
\frac{\mathrm{d} n_{S}}{\mathrm{~d} \ln k} & =2 \xi^{2}+\mathcal{O}\left(\alpha^{4}\right) \simeq 2 \frac{\alpha^{2}}{f^{4}}, \\
r & =16 \epsilon=8 \frac{\alpha^{2}}{f^{2}} \simeq 4 f^{2} \frac{\mathrm{d} n_{S}}{\mathrm{~d} \ln k} .
\end{aligned}
$$

We now see that a choice of a Planckian axion decay constant $f=1$ and $\alpha=0.1$ produces a red tilt $n_{S} \simeq 0.97$ and a sizable tensor-mode fraction $r \simeq 0.08$, while keeping the running of the spectral index $\mathrm{d} n_{S} / \mathrm{d} \ln k \simeq 0.02$ moderately small.

However, the embedding of this effective description into a string theory model dictates additional constraints on the parameters. For our purposes, the most relevant restrictions are on axion decay constants, which are the subject of Sec. III. Guided by this discussion, we choose to work with the fiducial bound $f \leqslant \sqrt{3} / 4 \pi$. Furthermore, we have to implement the observational constraints on $n_{S}$ and its running. These are $n_{S}=0.9603 \pm 0.0073$ and $\mathrm{d} n_{S} / \mathrm{d} \ln k=-0.0134 \pm 0.0090$ [2]. Using the constraints $f \leq \sqrt{3} / 4 \pi$ and $\mathrm{d} n_{S} / \mathrm{d} \ln k \lessgtr 0.01$, Eq. (9) dictates the bound $r \leqslant 7.6 \times 10^{-4}$, which in turn forces $n_{S} \simeq 1$, a value excluded by Planck at the level of $5 \sigma$.

However, $n_{S}<1$ is easily achieved by letting inflation start slightly above $\phi_{0}=\pi f / 2$. In this region of field space, $\eta$ takes the form

$$
\eta \simeq-\frac{\alpha}{f^{3}}\left(\phi_{N}-\phi_{0}\right)
$$

The measured value $n_{S}=0.9603$ dictates $\phi_{N} / \phi_{0} \simeq 1.18$.

Thus, we have consistently realized $r \simeq 7.6 \times 10^{-4}$ in a string-motivated setting. By contrast, the Lyth-bound estimate of Eq. (1), assuming constant $r$ and $\Delta N=60$, would give $r \simeq 1.4 \times 10^{-4}$. Hence, we gain a factor of $\simeq 5$ as compared to the Lyth approximation.

We can now compare this with the estimated precision of future cosmological probes. While the B-mode polarization search in the cosmic microwave background $(\mathrm{CMB})$ is expected to yield sensitivity of $r=(\mathrm{a}$ few $) \times$ $10^{-2}$ for Planck [54], the dedicated CMB polarization probe candidates CMBpol/EPIC [54] and PIXIE [55] can detect a tensor-to-scalar ratio down to $r \simeq 10^{-3}$. Even more promising is the analysis of the angular power spectra and weak lensing contribution to the $21 \mathrm{~cm}$ radiation, which can yield a B-mode detection down to $r \simeq 10^{-9}$ [56,57].

The potential of our hybrid axion inflation model flattens toward the end of the slow-roll regime. This means that the amplitude of curvature perturbations grows, implying the threat of primordial black hole overproduction. To evaluate the situation, we compare the curvature perturbations $\Delta_{\mathcal{R}}^{2} \propto H^{2} / \epsilon$ at the beginning and the end of inflation, $\Delta_{\mathcal{R}, N} / \Delta_{\mathcal{R}, c} \simeq \sqrt{\epsilon_{c} / \epsilon_{N}}$. Using Eqs. (5) and (8), we find 


$$
\frac{\Delta_{\mathcal{R}, N}}{\Delta_{\mathcal{R}, c}} \simeq 2 \exp \left(-\frac{60 \alpha}{f^{2}}\right) \sim 10^{-2} .
$$

Now, the most recent value [2] for the power spectrum is $\Delta_{\mathcal{R}}^{2} \equiv A_{S} \simeq 2.2 \times 10^{-9}$ at the fiducial scale $k=$ $0.05 \mathrm{Mpc}^{-1}$. This can be identified with our $\Delta_{\mathcal{R}, N}^{2}$. It can be compared to the most conservative primordial black hole production bound, which is $\Delta_{\mathcal{R}, c}^{2}<10^{-3}$ (see Refs. [58,59] and references therein). One finds $\Delta_{\mathcal{R}, N} / \Delta_{\mathcal{R}, c} \geq 10^{-3}$. Thus, in view of Eq. (11), our model is completely safe.

\section{STRINGY CONSTRAINTS}

String theory dictates additional constraints on the parameters, in particular on the axion decay constant [45]. Our focus will be on two types of axionic scalars: the imaginary parts of Kähler moduli and the real parts of complex structure moduli. Kähler axions descend from $p$-form potentials of the 10-dimensional theory upon dimensional reduction to four dimensions. To understand that complex structure moduli have anything to do with axions, recall that under mirror symmetry, the complex structure moduli space of type IIB string theory is mapped to the Kähler moduli space of type IIA. Thus, at large complex structure (which corresponds to large volume on the type IIA side), we expect an axionic shift symmetry to act on the complex structure moduli as well.

As a simple example, consider the axiodilaton $S=$ $i / g_{s}+C_{0}$, where $g_{s}$ is the string coupling and $C_{0}$ is the Ramond-Ramond (RR) zero-form potential. The Kähler potential for this modulus is $K=-\ln (-i(S-\bar{S}))$, giving rise to a kinetic term

$$
\mathcal{L} \supset K_{S \bar{S}}|\partial S|^{2} \supset\left(\frac{g_{s}}{2}\right)^{2}\left(\partial C_{0}\right)^{2} .
$$

The canonically normalized "would-be" inflaton is $\phi=$ $\frac{g_{s}}{\sqrt{2}} C_{0}$. The periodicity $C_{0} \rightarrow C_{0}+1$ of the RR zero-form field [60] implies a periodicity $\phi \rightarrow \phi+g_{s} / \sqrt{2}$, and hence $f=\frac{g_{s}}{\sqrt{2} 2 \pi}$. Therefore, already at the self-dual point $g_{s}=1$, the axion decay constant is much smaller than 1 . It decreases even further at weak coupling, $g_{s} \ll 1$.

From the F-theory perspective, $S$ is part of the complex structure moduli space of the fourfold. Therefore, we expect similar considerations to apply in the case of complex structure axions. The same is true for deformation moduli of D7-branes, as they are part of the complex structure of the F-theory fourfold as well. The analogs of $g_{s} \sim 1$ and $g_{s} \ll 1$ are generic and large imaginary parts of the complex structure moduli, respectively. We thus expect that the axion decay constant $f$ in Eq. (4) can take values as large as $f \sim 1 / 4 \pi$ at generic complex structure and $g_{s} \sim 1$.

In the example of Kähler axions [61-64], one obtains, via dimensional reduction, the term

$$
\mathcal{L} \supset \frac{1}{4 \pi} r^{i \alpha} c_{\alpha} \operatorname{tr}\left(F_{i} \wedge F_{i}\right),
$$

which displays the coupling of the axions $c_{\alpha}$ to the field strength of the gauge field living on a D7-brane (wrapping a four-cycle labeled by the index $i$ ). The $c_{\alpha}$ are the coefficients of an expansion of the RR four-form in terms of a basis of four-forms (labeled by the index $\alpha$ ) of the threefold. The integers $r^{i \alpha}$ arise from integrating the fourform labeled by $\alpha$ over the four-cycle labeled by $i$. Quantization of $\int \operatorname{tr}\left(F_{i} \wedge F_{i}\right)$ implies that the term (13) is trivial for integer values of $c_{\alpha}$ [64].

To read off the axion decay constant, one has to canonically normalize the axion, using the Kähler potential $K=$ $-2 \ln \mathcal{V}$. From here it is apparent that the axion decay constant typically scales with the inverse of some fourcycle volume, the precise value depending on the volume form. For example, for a scenario with one large fourcycle with volume ${ }^{7} \tau$ and corresponding Kähler modulus $T=\tau+i c$, such that $\mathcal{V} \sim(T+\bar{T})^{3 / 2}$, one finds [64]

$$
f=\frac{\sqrt{3}}{4 \pi \tau} .
$$

We take $f \leqq \sqrt{3} / 4 \pi$ as our fiducial value, corresponding to an Einstein frame four-cycle volume of unity. In the example of a compactification on a square $\left(T^{2}\right)^{3}$ and for $g_{s}=1$, this is the T-self-dual point. If one instead evaluates $f$ at the point where instanton corrections $\sim e^{-2 \pi \tau}$ become important, the bound on $f$ is generally weakened by a factor of $2 \pi$.

Note that for $f=\sqrt{3} / 4 \pi$ and $\mathrm{d} n_{S} / \mathrm{d} \ln k=0.01$, one has $\alpha=1.3 \times 10^{-3}$, which matches $\left.e^{-2 \pi \tau}\right|_{\tau=1}$ up to an $\mathcal{O}(1)$ factor. This is in very good agreement with our expectation that oscillatory potentials like Eq. (4) arise from instanton effects.

\section{CONCLUSIONS}

We have analyzed models representing a cross between axionic and hybrid inflation. They naturally produce a sufficient amount of $e$-foldings within a small field range. Moreover, the typical axionic modulation of the flat treelevel potential leads to a variation of $\epsilon$. This generates a significant tensor-mode contribution (up to $r \sim 10^{-3}$ ) in early inflation, where $\epsilon$ is sizable. The required number of $e$-foldings is accumulated later on, when $\epsilon$ approaches zero.

Obviously, the magnitude of curvature perturbations grows at high $\ell$. It would be interesting to look for observable consequences of this effect, e.g., along the lines of Refs. [65-68]. Finally, a more detailed analysis of the relevant stringy construction is clearly necessary and in progress [53].

\footnotetext{
${ }^{7}$ The (dimensionless) Einstein frame four-cycle volume $\tau$ is related to the string frame four-cycle volume as $\tau=g_{s}^{-1} \ell_{s}^{-4} \tau^{s}$, where $\ell_{s}=2 \pi \sqrt{\alpha^{\prime}}$ is the string length.
} 


\section{ACKNOWLEDGMENTS}

We would like to thank Stefan Sjörs and Timo Weigand for helpful discussions. This work was supported in part by the Impuls und Vernetzungsfond of the Helmholtz
Association of German Research Centres under Grant No. HZ-NG-603, by the Transregio TR33 "The Dark Universe," and by the National Science Foundation under Grant No. NSF PHY11-25915.
[1] P. Ade et al. (Planck Collaboration), arXiv:1303.5062.

[2] P. Ade et al. (Planck Collaboration), arXiv:1303.5082.

[3] A. D. Linde, Phys. Lett. 129B, 177 (1983).

[4] S. Kachru, R. Kallosh, A. Linde, J. Maldacena, L. McAllister, and S. P. Trivedi, J. Cosmol. Astropart. Phys. 10 (2003) 013.

[5] D. Baumann, A. Dymarsky, I. R. Klebanov, L. McAllister, and P. J. Steinhardt, Phys. Rev. Lett. 99, 141601 (2007).

[6] A. Krause and E. Pajer, J. Cosmol. Astropart. Phys. 07 (2008) 023.

[7] D. Baumann, A. Dymarsky, I. R. Klebanov, and L. McAllister, J. Cosmol. Astropart. Phys. 01 (2008) 024.

[8] N. Arkani-Hamed, H.-C. Cheng, P. Creminelli, and L. Randall, Phys. Rev. Lett. 90, 221302 (2003).

[9] K. Freese, J. A. Frieman, and A. V. Olinto, Phys. Rev. Lett. 65, 3233 (1990).

[10] N. Barnaby, E. Pajer, and M. Peloso, Phys. Rev. D 85, 023525 (2012).

[11] E. J. Copeland, A. R. Liddle, D. H. Lyth, E. D. Stewart, and D. Wands, Phys. Rev. D 49, 6410 (1994).

[12] A. D. Linde and A. Riotto, Phys. Rev. D 56, R1841 (1997).

[13] E. Silverstein and A. Westphal, Phys. Rev. D 78, 106003 (2008).

[14] L. McAllister, E. Silverstein, and A. Westphal, Phys. Rev. D 82, 046003 (2010).

[15] N. Kaloper and L. Sorbo, Phys. Rev. Lett. 102, 121301 (2009).

[16] M. Berg, E. Pajer, and S. Sjors, Phys. Rev. D 81, 103535 (2010).

[17] N. Kaloper, A. Lawrence, and L. Sorbo, J. Cosmol. Astropart. Phys. 03 (2011) 023.

[18] S. Dubovsky, A. Lawrence, and M. M. Roberts, J. High Energy Phys. 02 (2012) 053.

[19] A. Lawrence, Phys. Rev. D 85, 105029 (2012).

[20] G. D'Amico, R. Gobbetti, M. Schillo, and M. Kleban, Phys. Lett. B 725, 218 (2013).

[21] B. Shlaer, Phys. Rev. D 88, 130503 (2013).

[22] G. D’Amico, R. Gobbetti, M. Kleban, and M. Schillo, J. Cosmol. Astropart. Phys. 03 (2013) 004.

[23] D. H. Lyth, Phys. Rev. Lett. 78, 1861 (1997).

[24] L. Boubekeur and D. H. Lyth, J. Cosmol. Astropart. Phys. 07 (2005) 010

[25] M. U. Rehman, Q. Shafi, and J. R. Wickman, Phys. Lett. B 683, 191 (2010).

[26] C. Pallis and Q. Shafi, Phys. Lett. B 725, 327 (2013).

[27] Q. Shafi and J. R. Wickman, Phys. Lett. B 696, 438 (2011).

[28] I. Ben-Dayan and R. Brustein, J. Cosmol. Astropart. Phys. 09 (2010) 007.
[29] S. Hotchkiss, A. Mazumdar, and S. Nadathur, J. Cosmol. Astropart. Phys. 02 (2012) 008.

[30] J. L. Cook and L. Sorbo, Phys. Rev. D 85, 023534 (2012).

[31] L. Senatore, E. Silverstein, and M. Zaldarriaga, arXiv:1109.0542.

[32] N. Barnaby, J. Moxon, R. Namba, M. Peloso, G. Shiu, and P. Zhou, Phys. Rev. D 86, 103508 (2012).

[33] D. Carney, W. Fischler, E. D. Kovetz, D. Lorshbough, and S. Paban, J. High Energy Phys. 11 (2012) 042.

[34] E. Dimastrogiovanni and M. Peloso, Phys. Rev. D 87, 103501 (2013).

[35] T. Kobayashi and T. Takahashi, Phys. Rev. Lett. 110, 231101 (2013).

[36] A. D. Linde, Phys. Lett. B 259, 38 (1991).

[37] A. D. Linde, Phys. Rev. D 49, 748 (1994).

[38] J.D. Cohn and E. D. Stewart, Phys. Lett. B 475, 231 (2000).

[39] E. D. Stewart and J.D. Cohn, Phys. Rev. D 63, 083519 (2001).

[40] G. G. Ross and G. German, Phys. Lett. B 684, 199 (2010).

[41] G. G. Ross and G. German, Phys. Lett. B 691, 117 (2010).

[42] D. E. Kaplan and N. J. Weiner, J. Cosmol. Astropart. Phys. 02 (2004) 005.

[43] N. Arkani-Hamed, H.-C. Cheng, P. Creminelli, and L. Randall, J. Cosmol. Astropart. Phys. 07 (2003) 003.

[44] A. Avgoustidis, D. Cremades, and F. Quevedo, Gen. Relativ. Gravit. 39, 1203 (2007).

[45] T. Banks, M. Dine, P. J. Fox, and E. Gorbatov, J. Cosmol. Astropart. Phys. 06 (2003) 001.

[46] J.E. Kim, H.P. Nilles, and M. Peloso, J. Cosmol. Astropart. Phys. 01 (2005) 005.

[47] S. Dimopoulos, S. Kachru, J. McGreevy, and J. G. Wacker, J. Cosmol. Astropart. Phys. 08 (2008) 003.

[48] J. P. Conlon, J. Cosmol. Astropart. Phys. 09 (2012) 019.

[49] P. Binetruy and G. R. Dvali, Phys. Lett. B 388, 241 (1996).

[50] E. Halyo, Phys. Lett. B 387, 43 (1996).

[51] A. Hebecker, S. C. Kraus, D. Lüst, S. Steinfurt, and T. Weigand, Nucl. Phys. B854, 509 (2012).

[52] A. Hebecker, S. C. Kraus, M. Küntzler, D. Lüst, and T. Weigand, J. High Energy Phys. 01 (2013) 095.

[53] M. Arends, A. Hebecker, K. Heimpel, S. Kraus, D. Lüst, C. Mayrhofer, C. Schick, and T. Weigand (unpublished).

[54] D. Baumann et al. (CMBPol Study Team), AIP Conf. Proc. 1141, 10 (2009).

[55] A. Kogut et al., J. Cosmol. Astropart. Phys. 07 (2011) 025.

[56] K. Sigurdson and A. Cooray, Phys. Rev. Lett. 95, 211303 (2005).

[57] L. Book, M. Kamionkowski, and F. Schmidt, Phys. Rev. Lett. 108, 211301 (2012). 
[58] H. V. Peiris and R. Easther, J. Cosmol. Astropart. Phys. 07 (2008) 024.

[59] A. S. Josan, A. M. Green, and K. A. Malik, Phys. Rev. D 79, 103520 (2009).

[60] F. Denef, arXiv:0803.1194.

[61] J. P. Conlon, J. High Energy Phys. 05 (2006) 078.

[62] M. Goodsell, J. Jäckel, J. Redondo, and A. Ringwald, J. High Energy Phys. 11 (2009) 027.

[63] M. Cicoli, M. Goodsell, J. Jäckel, and A. Ringwald, J. High Energy Phys. 07 (2011) 114.
[64] M. Cicoli, M. Goodsell, A. Ringwald, M. Goodsell, and A. Ringwald, J. High Energy Phys. 10 (2012) 146.

[65] H. Tashiro and N. Sugiyama, Phys. Rev. D 78, 023004 (2008).

[66] J. Chluba, R. Khatri, and R. A. Sunyaev, arXiv:1202.0057.

[67] J. B. Dent, D. A. Easson, and H. Tashiro, Phys. Rev. D 86, 023514 (2012).

[68] J. Chluba, A. L. Erickcek, and I. Ben-Dayan, Astrophys. J. 758, 76 (2012). 